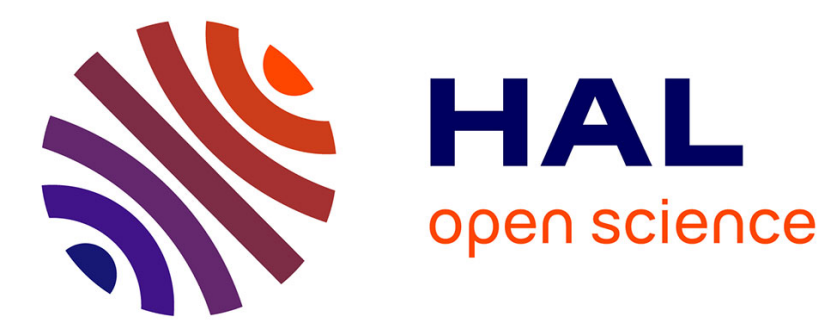

\title{
Le canal de Marius et les dynamiques littorales du golfe de Fos
}

\author{
Claude Vella, Philippe Leveau, Mireille Provansal, Jean-Marie Gassend, \\ Bertrand Maillet, Martine Sciallano
}

\section{To cite this version:}

Claude Vella, Philippe Leveau, Mireille Provansal, Jean-Marie Gassend, Bertrand Maillet, et al.. Le canal de Marius et les dynamiques littorales du golfe de Fos. Gallia - Archéologie de la France antique, 1999, Le Rhône romain, 56, pp.131-139. 10.3406/galia.1999.3250 hal-01913112

\section{HAL Id: hal-01913112 \\ https://hal.science/hal-01913112}

Submitted on 16 Jan 2020

HAL is a multi-disciplinary open access archive for the deposit and dissemination of scientific research documents, whether they are published or not. The documents may come from teaching and research institutions in France or abroad, or from public or private research centers.
L'archive ouverte pluridisciplinaire HAL, est destinée au dépôt et à la diffusion de documents scientifiques de niveau recherche, publiés ou non, émanant des établissements d'enseignement et de recherche français ou étrangers, des laboratoires publics ou privés.

\section{(ㅇ)(1) $\$$}

Distributed under a Creative Commons Attribution - NonCommercial - NoDerivatives| 4.0 


\title{
LE CANAL DE MARIUS ET LES DYNAMIQUES LITTORALES DU GOLFE DE FOS
}

\author{
Claude Vella, Philippe Leveau, Mireille Provansal \\ et la collaboration de Jean-Marie GaSsend, Bertrand MaIllet et Martine Sciallano
}

\begin{abstract}
Mots-clés. Canaux, commerce, delta du Rhône, Fos, hydrologie, ligne de rivage, Marius, navigation, sédimentologie, sources écrites.
Key-words. Canals, trade, Rhône delta, Fos, hydrology, littoral shoreline, Marius, navigation, sedimentology, written sources.

Résumé. Cet article fait le point des recherches concernant le canal creusé par Marius et les installations portuaires du golfe de Fos. Ces aménagements romains correspondent à un état de l'hydrologie du Rhône; les sources écrites et les données sédimentologiques concordent pour mettre cet événement en relation avec une modification de l'embouchure. Les variations de la ligne de rivage dans le golfe de Fos rendent compte des difficultés de la recherche archéologique portant sur un secteur où une succession d'avancées et de reculs affecte la lecture des structures archéologiques.
\end{abstract}

\begin{abstract}
This paper sums up researches concerning the canal built by Marius and harbour installations in the gulf of Fos. These roman developments have relevance to a state of the hydrology of the Rhone. Written sources and sedimentology data are in concordance to associate this event with the change of the mouth, the variations of the shoreline in the gulf explain difficulties in archaeological research in an area where repeated rise and fall affects the reading of archaeological features.
\end{abstract}

Pour faciliter l'entrée du Rhône, Marius fit creuser un canal à l'est du delta. Ouvert en 102 avant notre ère, cet ouvrage pose toujours un irritant problème aux archéologues. Connu depuis le XVIII ${ }^{e}$ s., l'emplacement de Fossae, le port qui se développa à son débouché maritime et lui doit son nom, a été confirmé par des découvertes sous-marines effectuées de part et d'autre de la Pointe de Saint-Gervais (Gateau, 1996, p. 169-188). Néanmoins la localisation du canal lui-même nous échappe encore. La construction d'un grand complexe portuaire et industriel n'en est pas la seule cause. Les vestiges archéologiques et les aménagements recherchés ont subi le jeu complexe des dynamiques génératrices des embouchures et des traits de côtes successifs du delta d'un des grands fleuves européens.

L'étude des aménagements portuaires est donc étroitement dépendante des connaissances de l'histoire holocène du littoral du golfe de Fos, qui n'a pris son appa- rence actuelle que très récemment. Jusqu'à ces dernières années, les recherches avaient porté surtout sur la Camargue et la partie occidentale du delta (Kruit, 1955 ; Duboul-Razavet, 1956 ; Bazile, Bazile-Robert, 1974 ; L'Homer et al., 1981) et ne s'intéressaient au secteur oriental que de manière marginale. De nouveaux travaux permettent de reprendre sur une base géo-archéologique un problème que les archéologues posaient à partir d'un état maintenant dépassé de la recherche sur le littoral.

\section{LES GRANDES PHASES \\ DE L'ÉVOLUTION DU TRAIT DE CÔTE DANS LE GOLFE DE FOS}

Le golfe de Fos est limité à l'est par le cailloutis de Crau dont le toit forme le substrat résistant et la topo- 


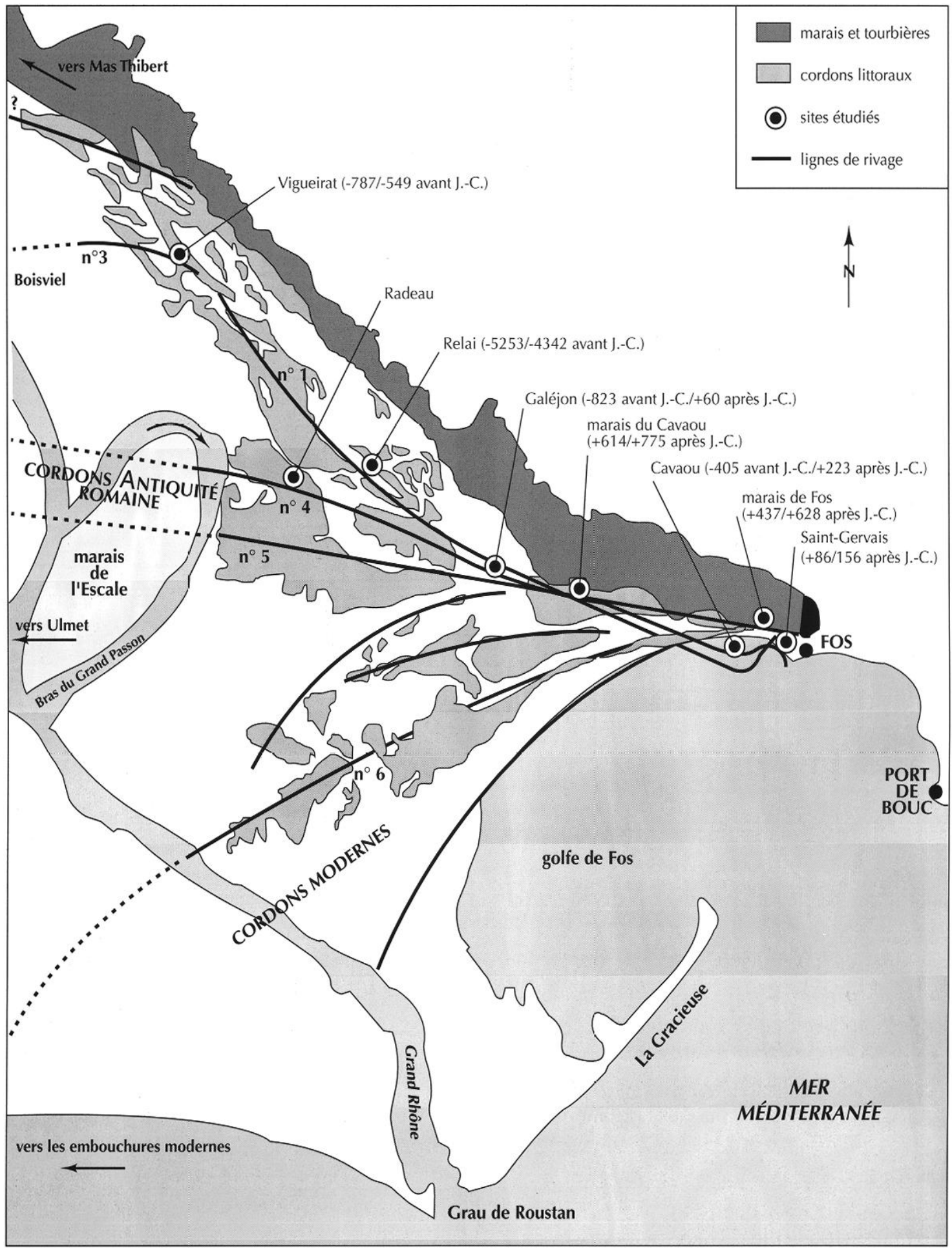

Fig. 48 - Positions du trait de côte (1-6) et sens de la progradation du littoral dans l'anse de Saint-Gervais.

graphie initiale sur lesquels a transgressé la mer et où se sont accumulés des dépôts qui s'amincissent vers l'est à mesure que l'on s'éloigne du delta. Les dynamiques qui ont donné naissance à ses traits de côtes successifs sont liées en premier lieu à la montée générale du niveau marin d'origine climato-eustatique. Sa vitesse se ralentit 
progressivement depuis 4000 ans environ (Laborel et al., 1994) mais, dans ce golfe, elle se combine à des mouvements verticaux négatifs du sol d'origines diverses (Vella et al., 1998). La formation du trait de côte est également commandée par un bilan sédimentaire qui enregistre la combinaison de trois facteurs : les variations de la charge sédimentaire du Rhône, la proximité d'un de ses exutoires et la puissance de la dérive littorale.

La chronologie des déplacements du trait de côte résumée ici a été établie à partir de carottages sédimentaires effectués sur des transects perpendiculaires au littoral prolongés par des sondages en terre ferme. Pour ce secteur, les données ont été collectées dans l'anse de Saint-Gervais et sur la rive gauche du fleuve, de MasThibert à Fos (fig. 48). Les caractères sédimentologiques et faunistiques des sédiments meubles permettent en effet de déterminer s'ils ont été déposés sur la terre ferme (tourbières), dans une lagune ou en mer ouverte. L'observation des concrétions organogènes sous-marines (Mesophyllum lichenoïdes), qui colonisent le cailloutis de Crau, complète l'information. Une trentaine de dates radiocarbone ont été effectuées sur des matériaux très divers - tourbes, posidonies, bois, coquilles marines, coralligène - avec des fourchettes d'incertitude de l'ordre du sic̀cle ; aucunc n'a ćté ćcartéc. Pour la concordance avec les faits historiques, elles sont ici indiquées en années calibrées (calendrier solaire) de l'ère chrétienne (années cal. avant ou après J.-C.). L'ensemble des indicateurs permet d'établir une position du trait de côte définie avec précision lorsque les carottages ont été effectués au sein des cordons littoraux. Elle est évaluée par défaut lorsque les données correspondent à la mer ouverte (épaves, coralligène) ou aux milieux juxtalittoraux (lagunes et tourbières isolées de la mer par des cordons).

Dans l'état actuel de la recherche, les différentes positions ont été regroupées en six phases de durée inégale, dont la dernière correspond à la période moderne (fig. 48). La phase $\mathrm{n}^{\circ} 1$ est datée de la fin du $\mathrm{VI}^{\mathrm{e}}$ et du $\mathrm{V}^{\mathrm{e}}$ millénaire avant notre ère. Entre celle-ci et la constitution des cordons de l'Antiquité romaine (phase $\mathrm{n}^{\circ} 4$ ), on distingue une phase $\mathrm{n}^{\circ} 2$ correspondant au début des Âges des Métaux (milieu du III ${ }^{e}$ millénaire) et une phase $\mathrm{n}^{\circ} 3$ qui est le rivage du Bronze final et du début de l'Âge du Fer. Elles peuvent s'analyser comme il suit. Entre 5200 et 2500 avant J.-C. environ, une succession de plusieurs générations de cordons marque un recul régulier du trait de côte, sauf vers 3690 cal. avant J.-C. (Ly 8150 ) et jus- qu'en 1928 cal. avant J.-C. (Ly 5249), moment où une tourbière s'installe et prograde, même légèrement, sur le domaine marin. Au niveau du Grand Rhône actuel, une échancrure dessine un golfe assez profond. Puis à l'est du delta, le rivage commence à se régulariser, mais la position des embouchures reste hypothétique. Bien que le littoral de Saint-Gervais enregistre les premières arrivées massives de sables rhodaniens, la sédimentation littorale est encore dominée par les galets de Crau (cordon de Boisviel). Dans le delta, un nouveau bras se dessine à l'est de celui de Saint-Ferréol, préfigurant le bras d'Ulmet plus proche de Fos (L'Homer et al., 1981; ArnaudFassetta, 1998) qui, au Bronze final, enregistre des crises détritiques sableuses.

\section{L'ÉVOLUTION DU LITTORAL À PARTIR DU II S. AVANT J.-C.}

Le creusement d'un canal par les Romains à la fin de l'Âge du Fer s'inscrit donc dans une évolution tendant à combler progressivement le golfe. Alors que, vers le sud, celui-ci est fermé par des cordons en éventail, l'évolution de détail est plus complexe dans le secteur de SaintGcrvais. Lcs sondages cffcctućs pròs du Galćjon ct lcs corps sédimentaires cartographiés sur la carte géologique situent le littoral antique (fig. $48, n^{\circ} 4$ ) à proximité du méandre du Grand Passon. Depuis la constitution du trait de côte correspondant à la fin de l'Âge du Bronze et au début de l'Âge du Fer (787 et 549 cal. avant J.-C., Ly 8023), l'accrétion est d'environ $2 \mathrm{~km}$, ce qui correspond à une vitesse de comblement du golfe de $7 \mathrm{~m}$ par an. L'orientation ouest-nord-ouest/est-sud-est du cordon est probablement induite par les houles de ouest-sudouest résultant de la réfraction des houles de mistral, comme aujourd'hui. Le tracé plus rectiligne de la côte permet une efficacité accrue de la dérive littorale, qui véhicule désormais massivement les sédiments sableux rhodaniens vers le sud-est. Dans le secteur de SaintGervais, un bilan sédimentaire largement positif favorise une progradation du littoral de $400 \mathrm{~m}$ environ.

C'est sur ce nouveau cordon qu'ont été édifiées des installations - attribuées à la station romaine de Fossae dont les vestiges immergés ont été découverts par les équipes de plongeurs depuis 1946 dans l'anse de SaintGervais. Ces données archéologiques complètent l'information sur l'âge et la position du trait de côte. Il s'agit 


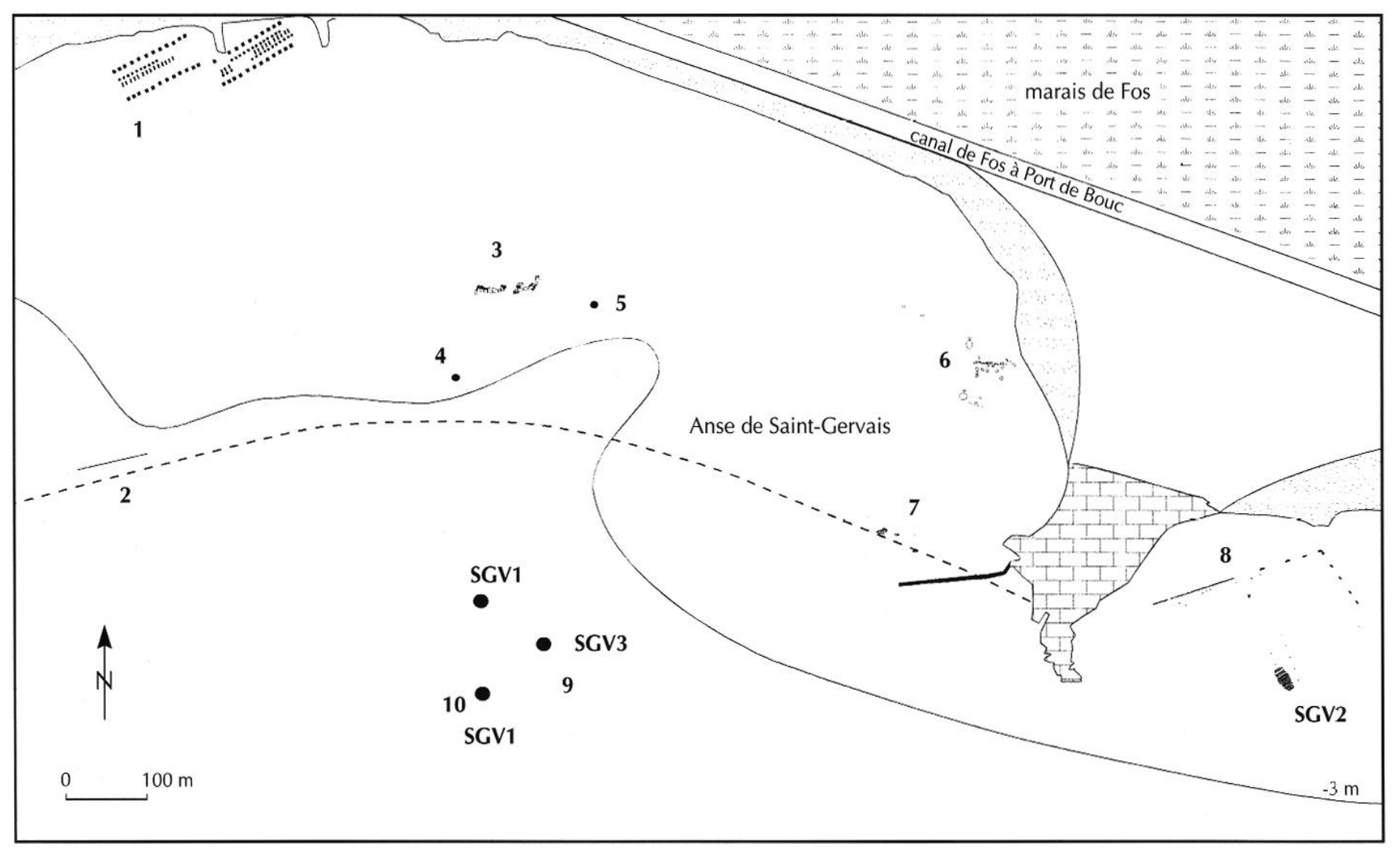

Fig. 49 - Position des vestiges archéologiques dans l'anse de Saint-Gervais : 1, structures quadrangulaires interprétées comme hangars/navalia (Gassend, Maillet, 1990) ; 2, nécropole (Liou, Monguilan, 1977) ; 3, mur (J.-M. Gassend) ; 4, palissades ; 5, pierres et pieux liés par du "bitume "; 6, alignements de pierres, poutres et tonneaux isolés (Sciallano, Leffy, 1992); 7, pieux et pierres (Dovis, 1991); 8, pieux et épave Saint-Gervais 2 du VII' s. après J.-C. (Jézégou, 1983) ; 9, épaves Saint-Gervais 1 et 3 datées de 139-140 après J.-C. et du IIIF s. après J.-C. (Liou, Gassend, 1990).

soit d'épaves en mer ouverte (Long, 1994) (fig. $49, \mathrm{n}^{\text {os }} 8$ et 9), soit de fondations de bâtiments et blocs d'architecture (Liou, 1987). Un ensemble remarquable a été localisé par prospections aériennes sur la plage du Cavaou, dans une zone recouverte actuellement par 1 à $2 \mathrm{~m}$ d'eau (Monguilan, 1977). Deux structures quadrangulaires de grandes dimensions (110 m x $36 \mathrm{~m}$ ) ont été reconnues. Elles sont définies par des dés de pierre disposés selon des alignements. Dans ces dés ont été évidées des cavités d'encastrement de poteaux, suggérant qu'il s'agit de vastes hangards (Gassend, Maillet, 1990) (fig. 49, $\mathrm{n}^{\circ} 1$ ). En outre, les plongeurs du DRASM ont identifié une vingtaine de stèles et d'autels funéraires disséminés sur un vaste espace; ces blocs paraissent ordonnés selon deux axes perpendiculaires d'orientation nord-sud et estouest (Liou, Monguilan, 1977) (fig. 49, $\mathrm{n}^{\circ}$ 2). L'étude archéologique en cours se heurte aux difficultés inhérentes au milieu marin ; ces structures ont dû en particu- lier subir les effets de soutirages propres aux milieux sédimentaires meubles. Les stèles et autels appartiendraient à une nécropole antique. Les structures allongées pourraient correspondre à des hangars à bateaux (navalia). L'hypothèse d'une zone portuaire au débouché du canal romain n'est pas retenue ici pour des raisons de méthode afin d'éviter un raisonnement circulaire : admettre qu'il s'agit d'installations portuaires suppose, en effet, résolu le problème archéologique discuté ici. Retenons donc que ces données donnent un terminus : le cordon sur lequel est positionnée la nécropole était achevé antérieurement au positionnement des monuments funéraires, soit au I ${ }^{\mathrm{er}} \mathrm{s}$. de notre ère. En outre, l'ensemble permet de reconnaître avec certitude un secteur émergé et de situer la côte à l'époque de Fossae.

Une stabilisation du niveau marin relatif peut expliquer l'avancée du littoral à la fin de l'Âge du Fer. Mais l'hypothèse la plus probable est un accroissement du 
bilan sédimentaire. Les sources écrites expliquent la construction du canal précisément par une obstruction partielle de l'embouchure par les sédiments ( $c f$. infra). Au même moment à Arles et dans le delta, les études sédimentologiques montrent un accroissement des débits liquides et solides du Rhône (Arnaud-Fassetta, 1998 et Provansal et al., supra, p. 27-28). L'ouverture d'une nouvelle embouchure à l'est permettrait d'expliquer le renforcement du flux sédimentaire dans le secteur de Saint-Gervais; la finesse des sables qui arrivent à Fos impliquant un long transport par la dérive littorale, elle ne saurait être située trop près de Fos. Il faut donc abandonner, au moins pour l'Antiquité, l'hypothèse longtemps admise d'un bras du Rhône débouchant au niveau du Galéjon, dont l'étang aurait été le vestige (Duboul-Razavet, 1956; L'Homer et al., 1981) (fig. 50b).

Au cours de l'Antiquité tardive et du Haut Moyen Âge, un recul important affecte ce secteur du littoral. Dans l'anse de Saint-Gervais, entre le VIII et le $\mathrm{X}^{\mathrm{c}} \mathrm{s}$. (Ly 8447, 7590, 7861), le rivage s'établit à $400 \mathrm{~m}$ au nord de la côte actuelle, dans la tourbière d'eau douce jusqu'alors épargnée par les intrusions marines. Il se prolonge à l'ouest, dans le marais du Cavaou, où il est daté cntrc 614 ct 775 cal. (Ly 8026) (fig. 48, cordon $\mathrm{n}^{\circ} 5$ ). Ce recul peut s'expliquer par une diminution des apports sédimentaires, par une augmentation de l'énergie marine ou par une variation locale du niveau marin due à des mouvements négatifs du sol. Cette dernière hypothèse est vérifiée par la déformation des paléoniveaux marins de l'anse de Saint-Gervais (Vella, Bourcier, 1998 ; Morhange et al., 1998). Les apports liés à la crise sédimentaire et hydrologique mise en évidence pour cette époque dans le delta et à Arles (ArnaudFassetta, 1998) ne suffisent donc pas à compenser la montée accélérée du niveau marin relatif. Ce recul fournit du même coup un terminus ante quem aux structures et aux hangars : il ne peut s'agir de constructions liées au maintien de l'activité portuaire attestée jusqu'au VII et au VIII ${ }^{\mathrm{e}}$ s. Lorsque, sur la colline proche, naît le bourg castral de Fos dont les premières mentions remontent au $\mathrm{X}^{\mathrm{c}}$ s. (Gateau, 1996, p. $178, \mathrm{n}^{\circ} 41$ ), une partie du site antique est sous l'eau.

La disposition des cordons est en relation avec la position des embouchures. Leur orientation est-ouest et leur faible courbure semblent indiquer que l'embouchure à laquelle ils se raccordent s'est déplacée vers le sud. Resté très actif le bras d'Ulmet (Arnaud-Fassetta, 1998) peut avoir alors joué un rôle important dans la régularisation du trait de côte. À l'époque moderne, la création du bras du Grand Passon, puis celle du Bras de Fer rapprochent de Fos l'embouchure principale, ce qui conduit les cordons à prendre une direction ouest-sudouest/est-nord-est (fig. 48, $\mathrm{n}^{\circ} 6$ ). Les cartes dont on dispose alors confirment leur angle d'incidence avec les embouchures, qui prennent de nouveau une forme digitée. Le dessin actuel du golfe apparaît nettement (Caritey, 1995). Sa position abritée limite les apports sédimentaires qui ne prennent de l'importance que durant la période de la crise climatique du Petit Âge Glaciaire et, en particulier, à l'époque moderne, quand une brèche ( crevasse ") ouverte dans le méandre de I'Escale assure une communication entre le fleuve et le golfe par l'étang du Galéjon.

$\mathrm{Au}$ total, l'histoire qui se dessine contraint à réorienter les recherches portant sur le canal romain. En se fondant sur la topographie du golfe au siècle dernier, M. Clerc avait proposé le tracé admis depuis (Clerc, 1906, p. 76-81) (fig. 50a) : les ingénieurs de Marius auraient réalisé une liaison entre des étangs et des lagunes qui se développent à l'est du delta, au pied de la Crau ; l'étang du Galéjon aurait constitué le débouché des Fosses Mariennes (Desjardins, 1876, p. 19-45 ; Gérin-Ricard, 1933, p. 363-366 ; Grenier, 1934, p. 493-509 ; Benoit, 1955 , p. 134). Les données paléoenvironnementales présentées ici infirment cette hypothèse : les carottages ont montré qu'une trace signalée par Max Guy (communication orale) correspondait en réalité à un paléorivage, dont le souvenir avait été conservé par une lagune figurant encore sur la carte de Cassini sous la forme d'un appendice de l'étang du Galéjon. L.-A. Constans cherchait l'exutoire du canal près d'un méandre plutôt que dans un golfe. Il interprétait ce que nous savons (et que l'on savait!) être un ancien cordon littoral comme le remblai d'un canal et cherchait donc à le positionner dans une zone, dont nous savons maintenant qu'elle n'était pas encore complètement émergée (Constans, 1921, p. 199) : le littoral antique se trouve en arrière de celui qu'il proposait, du moins pour la partie aval du canal. Le canal devait relier le bras d'Ulmet à un golfe situé au nord-ouest de Fos. Mais les mouvements négatifs du sol et surtout l'érosion littorale ont pu entraîner la disparition de son débouché dans le golfe. 


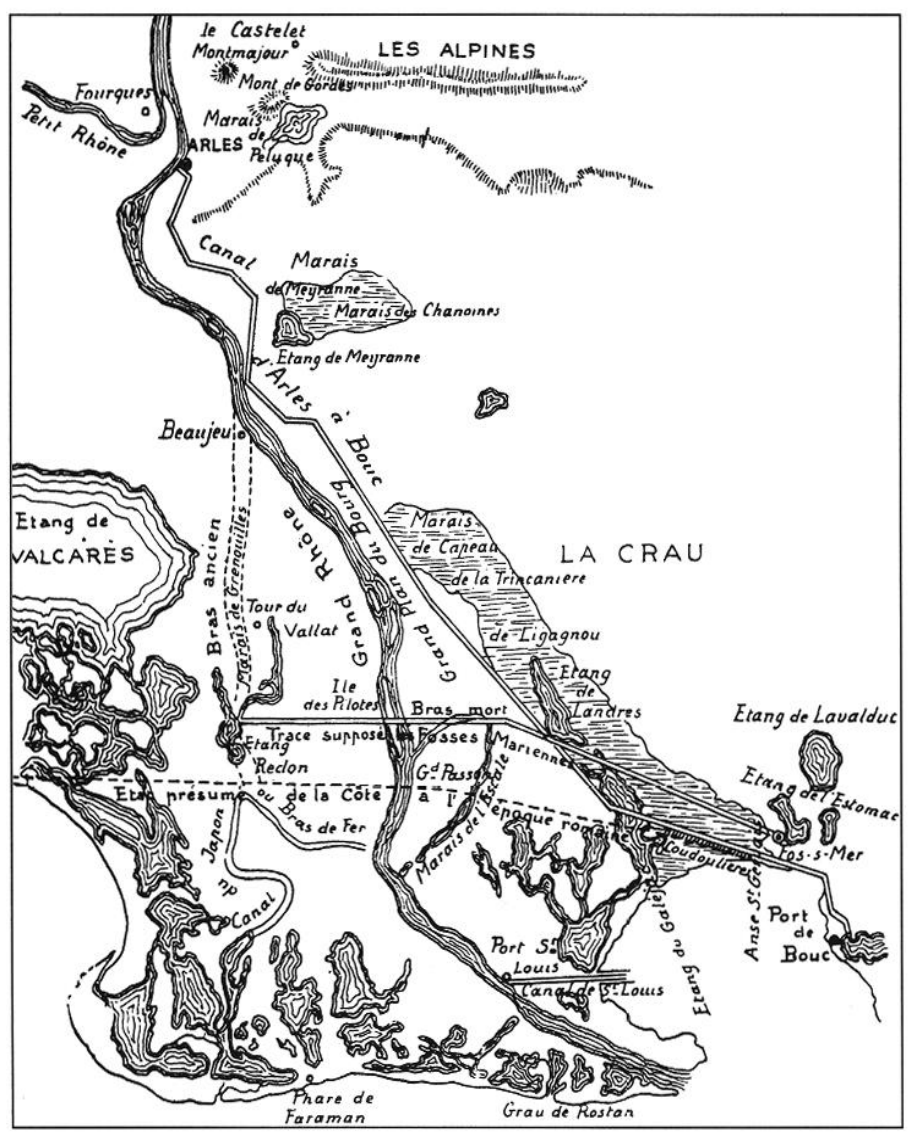

a

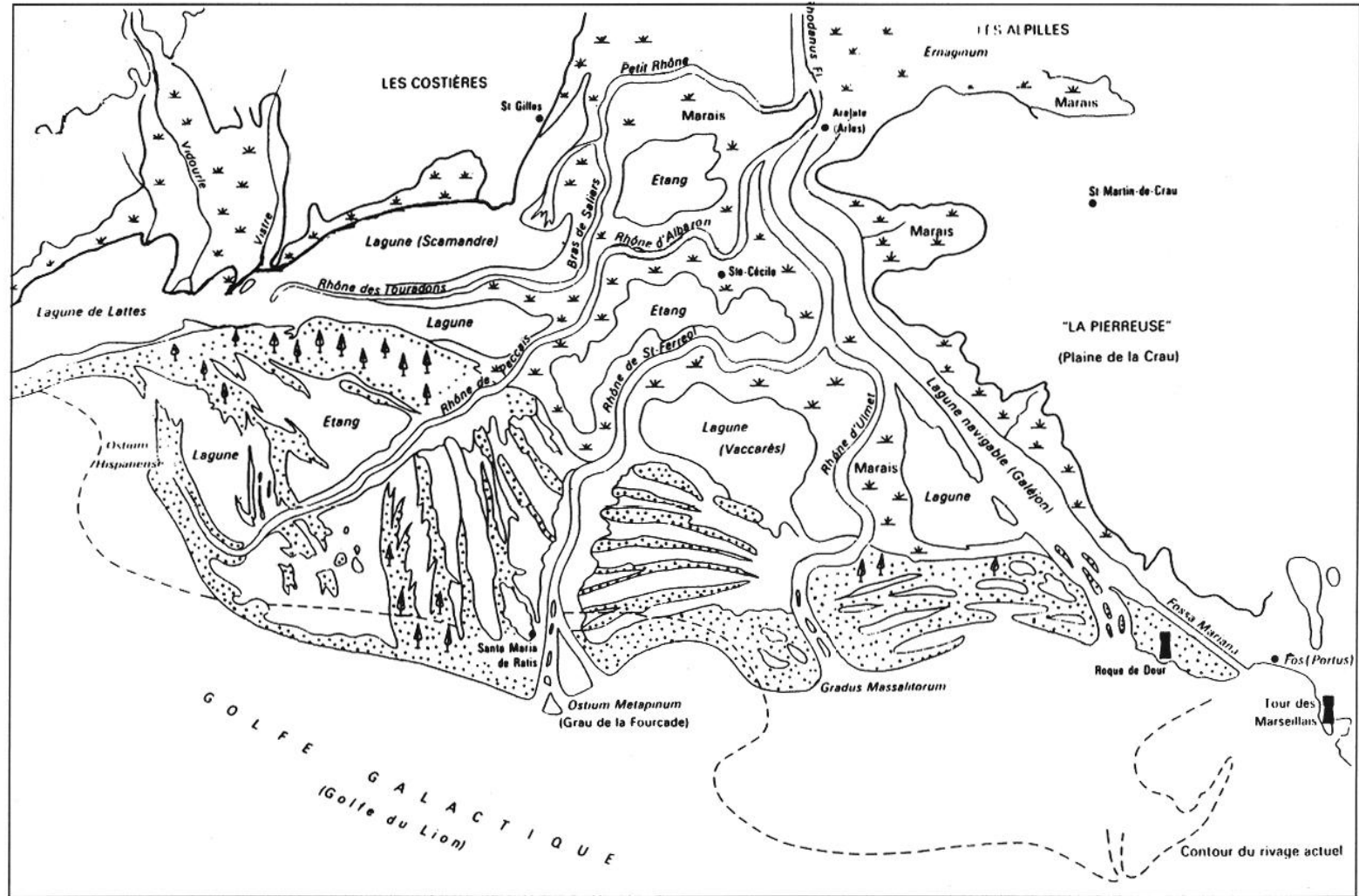

b

Fig. 50 - Localisations hypothétiques des Fosses Mariennes : a, d'après Crenier, 1934, fig. 164 (d'après Clerc, 1906 et Constans, 1921) ; b, d'après L'Homer, 1987 (notice de la carte géologique d'Arles), carte de l'organisation des cours du Rhône à la fin de l'époque romaine, cf. Cabot, 1991, p. 12. 


\section{LA CONSTRUCTION DU CANAL DE MARIUS ET LES DYNAMIQUES DU RHÔNE}

À ce moment du raisonnement, il est possible d'utiliser des sources écrites. Leurs indications sont cohérentes avec les données sédimentologiques et archéologiques présentées. Cette cohérence permet de préciser l'histoire de cette partie du delta en datant l'apparition de la bouche dont il a été question. En effet, si Pomponius Méla se contente d'écrire que "le canal de Marius déverse dans un lit navigable une partie de ce fleuve » ( $D e$ situ orbis, 2, 5, 77), Strabon et Plutarque sont explicites dans deux passages qu'il convient de reproduire.

Strabon (Géngraphie, IV, 1, 8) : « Voyant que les dépôts d'alluvions aveuglaient ces embouchures et rendaient leurs accès difficiles, Marius fit creuser un nouveau canal qui devait accueillir la plus grande partie des eaux du fleuve et en fit don aux Massaliotes pour les récompenser de leur bravoure [...]. Néanmoins l'accès demeure difficile aux navires à cause de la force du courant, de l'accroissement des dépôts alluviaux et du niveau du pays qui est si bas qu'on n'en distingue pas la côte, même de près, les jours de mauvais temps. Aussi les Massaliotes ont-ils édifié des tours servant de point de repère [...]. » (trad. Lasserre).

Plutarque (Vie de Marius, XV) : « Les embouchures du Rhône, à cause du refoulement opéré par la mer, recevant quantité de limon et de sable que la vague comprime en boue épaisse, offraient aux navires chargés de blé une entrée difficile, laborieuse et étroite. Marius [...] creusa un grand canal, y fit passer une bonne partie du fleuve et le conduisit à un endroit commode du rivage, là où il est profond, capable de recevoir de grands navires et en même temps plat et mettant l'embouchure à l'abri des vagues. » (trad. B. Lazarius).

Plutarque et Strabon mettent explicitement la décision de Marius en relation avec une gêne pour la navigation entrainée par une élévation de la barre. On n'en connât évidemment pas le niveau à la fin du II ${ }^{e} \mathrm{~s}$. avant J.-C. Mais, compte tenu du tirant d'eau des navires de l'époque qui est compris entre 1,2 et $3 \mathrm{~m}$ (Pomey, 1992 ; Roman, 1997), elle devait être plus basse que la barre d'embouchure actuelle qui se situe en été autour de 2,5 m (port autonome de Marseille, 1993). La connaissance des processus naturels d'édification des barres sableuses, qui constituent un danger majeur pour la navigation au contact de la mer et du fleuve, n'a été précisée qu'en 1937 (François, 1937). À partir du XVIII s., les auteurs débattent de leur origine marine ou fluviale. La justesse de l'observation de Plutarque est remarquable : il fait la part respective de la mer et du fleuve dans leur formation. Cette remarque contribue à valider les indications qu'il donne.

\section{LE GOLFE DE FOS ET LES EMBOUCHURES DU RHÔNE}

Le creusement du canal correspond à un état du delta ; son maintien en fonction était lié aux conditions de la navigation dans le delta, soit à l'histoire des embouchures du fleuve. Les sources antiques varient considérablement quant à leur nombre antérieurement au début de notre ère. Un vers des Argonautes d'Apollonios de Rhodes (IV, 634) (III ${ }^{e}$ s. avant J.-C.) fait allusion à sept embouchures, alors qu'elles seraient au nombre de six selon Possidonios et de cinq d'après Diodore de Sicile ( 5 ,

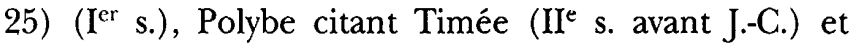
Festus Avienus $\left(\mathrm{IV}^{\mathrm{e}} \mathrm{s}\right.$.). Cette variation est considérée comme une preuve de l'incohérence des sources. Pour notre part, plutôt que de leur refuser toute crédibilité, nous préférons suivre $E$. Desjardin, pour qui ces contradictions notent des états successifs d'un delta en évolution (Desjardin, 1876, p. 196-231), et rechercher la cohérence des sources écrites avec les données archéologiques et sédimentologiques (fig. 51). Nous relevions la pertinence des observations de Plutarque sur la barre du Rhône. Strabon fait également preuve d'une bonne perception du milieu deltaique dans un passage concernant « une de ces lagunes qu'on appelle stomalimné», où il explique que " certains auteurs ont compté cette lagune au nombre des bouches du Rhône, notamment ceux qui parlent de sept embouchures " (Strabon, Géographie, IV, 1, 8) ; effectivement, des graux ont pu être assimilés à un bras du fleuve.

Dans cette perspective, un examen attentif des sources permet de placer dans les années 125 avant J.C. le moment où le Rhône passe de deux à trois embouchures. Corrigeant Timée qui aurait tiré son information de Pythéas (IV s.) ou d'un périple ancien correspondant à un état antérieur du delta non examiné ici, Polybe (II ${ }^{e}$ s.) écrit « au sujet des bouches du Rhône [qu'il n'y en a] pas cinq mais seulement deux [...]. » Cette indica- 


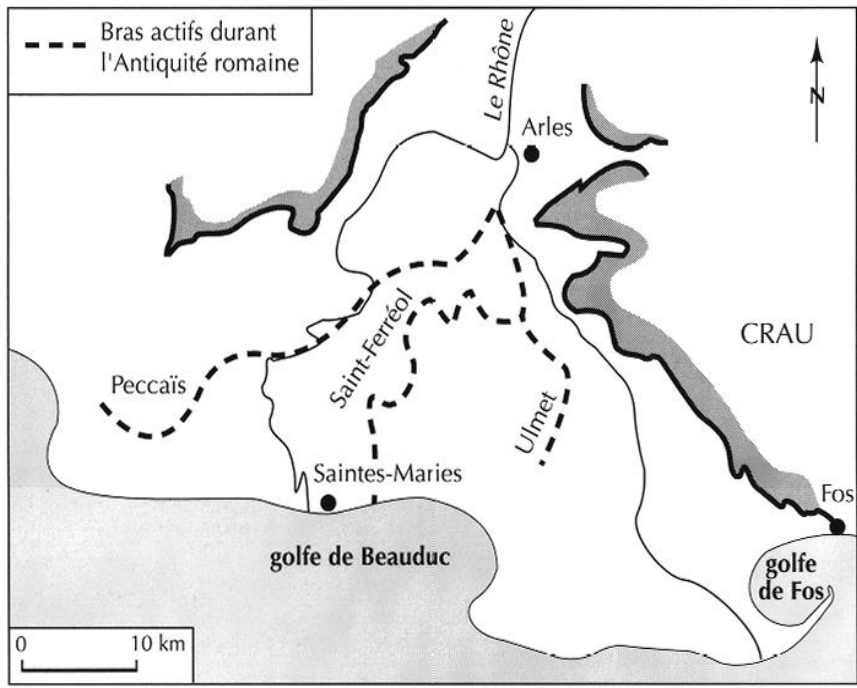

Fig. 51 - Schéma de localisation, d'après les notices des cartes géologiques 1/50 000 BRGM (d'après L'Homer, 1987 : Arles ; 1993 : Grau-du-Roi).

tion vaut pour le troisième quart du $\mathrm{II}^{\mathrm{c}} \mathrm{s}$. avant notre ère. Tite-Live (début $\mathrm{I}^{\mathrm{er}} \mathrm{s}$.) se contente de dire que « le fleuve se divise en plusieurs bras avant de se jeter dans la mer » (XXI, 26, 21, 3). Mais, vers le changement d'ère, Strabon rapporte que le Rhône a trois embouchures (Géographie, IV, 1, 8, C 208) et rappelle qu'Artémidore (vers les années 100 avant J.-C.) en comptait déjà trois. Le même chiffre est donné par Pline (milieu I ${ }^{\text {er }} \mathrm{s}$.) qui précise qu'à son époque, soit au $\mathrm{I}^{\mathrm{cr}} \mathrm{s}$. après J.-C., la bouche Massaliotique est la plus large (Pline, Histoire naturelle, III, 5). Au II ${ }^{\mathrm{e}}$ s., Ptolémée ne connaît que deux embouchures. Mais trois bouches sont figurées sur la Table de Peutinger. De même au Ve s., le poète Martianus Capella donne le nom de trois bouches (De nuptiis Philogiae et Mercuri libri, VI, 686). La mosaïque d'Ostie, où est figurée l'embouchure d'un fleuve à trois bouches précédée d'un pont de bateau, ne peut être utilisée ici puisque cette configuration permet précisément d'identifier le Rhône plutôt qu'un autre fleuve.

L'évolution serait donc la suivante : au IV $\mathrm{s}$. avant J.-C. (Pythéas), cinq embouchures auraient fonctionné ; au milieu du II ${ }^{\mathrm{e}}$ s., il n'y en aurait eu plus que deux (ce qui est le chiffre actuel); vingt ans après, une nouvelle embouchure serait (ré)apparue. Entre la fin du $\mathrm{II}^{\mathrm{c}} \mathrm{s}$. (Artémidore) et la fin de l'Antiquité, le chiffre serait demeuré à trois. Il est probable que ces embouchures soient celle du bras prolongeant le Rhône d'Albaron (L'Homer et al., 1981 ; 1993), celle du bras de Saint-
Ferréol actif durant toute l'Antiquité (Arnaud-Fassetta, 1998) et celle du bras d'Ulmet (L'Homer et al., 1981). Cette dernière est probablement la plus proche de Fos. Reste à rendre compte des indications fournies par Ptolémée qui n'indique que deux embouchures dans son Guide géographique (II) ? Commet-il une erreur ? On peut le penser car il place Fossae Marianae à l'ouest du delta. Pourtant le littoral était la partie la mieux connue et fournissait les principaux repères (Duval, 1971, p. 453-454).

\section{LA STATIO DE FOSSAE MARIANAE}

Le développement d'une agglomération maritime, la statio de Fossae Marianae, est lié aux dynamiques deltaïques qui sont à son origine. Une station douanière du Quarantième des Gaules - impôt institué au $\mathrm{I}^{\mathrm{er}} \mathrm{s}$. - a pu y exister (France, Hesnard, 1995, p. 85), mais les documents qui parlent de Fossae sont postérieurs de plus de deux siècles à la construction du canal auquel elle doit son nom. Le premier est l'Itinéraire Maritime dit Périple de Rome à Arles (Itinerarium portuum vel positionum navium ab urbe Arelato usque) qui la cite comme portus, terme qui se distingue de positio, mouillage. La statio figure également sur une vignette de la Table de Peutinger, copie médiévale d'un document antique. Ce document lui donne même une grande importance : la vignette qui la représente est égale à celle d'Ostie. Fos serait donc l'avant-port d'Arles et le port du Rhône, comme Ostie est le port de Rome. Cependant, détail essentiel, A. Grenier a montré que le canal ne figure pas sur la vignette : le trait qui relie Fos à Arles est une route (Grenier, 1934, p. 501 note 4). Ajoutons qu'il convient de ne pas passer trop rapidement de l'iconographie à l'économie. La vignette d'Ostie représente un port et, à l'entrée, un phare ; celle de Fossue Murianue ne figure pas le port. Le canal reliant Rome à Ostie faisait de ce port un point de rupture de charge pour les navires de ravitaillement. D'après l'Itinéraire Maritime, qui ne fait pas non plus état du canal, pour gagner Arles les navires remontent le fleuve en entrant dans le delta par le Gradus Massalitanorum, 18 milles à l'ouest de Fossae. De plus, la notion d'avant-port d'Arles ne rend pas compte de toute l'activité de Fos à la fin de l'Antiquité : les recherches actuelles montrent que la région des étangs connaît alors un fort peuplement (Trément, 1999). L'agglomération a pu récupérer les fonctions exercées autrefois 
par Saint-Blaise. Cette activité régionale et le transbordement d'une partie des cargaisons sur des barques empruntant le canal demeuré en usage pour celles-ci (seulement et non plus pour les navires) (Grenier, 1934, p. 505) expliqueraient la présence d'un matériel archéologique abondant.

L'évolution suivante peut être proposée : avec l'apaisement de la crise du fleuve, la barre a pu devenir à nouveau franchissable, rendant progressivement le canal inutile à partir du $\mathrm{I}^{\text {er }}$ s. de notre ère. Depuis le $\mathrm{II}^{\mathrm{e}}$ s., les bateaux ont pu de nouveau entrer dans le Rhône par le Gradus Massalitanorum comme l'indique l'Itinéraire Maritime. Ce gradus est également mentionné par Ammien Marcellin au IV s. : "Spumeus Rhodanus Gallico mari incorporatur per patulum sinum quem vocant Ad Gradus, $a b$ Arelate octavo decimo ferme lapide dispartum " (Histoire, $\mathrm{XV}, 11,18)$. Ce texte a donné lieu à deux interprétations. M. Clerc traduisait : « le Rhône écumeux s'unit à la mer (de Gaule) par une large baie qu'ils appellent Ad Gradus, distante d'Arles d'environ 18 milles » $(26,25 \mathrm{~km})$. Observant que la distance était peu vraisemblable car elle obligeait à situer le rivage trop en amont, L.-A. Constans a proposé de traduire : "Le Rhône écumeux mêle ses eaux à celle de la mer de Gaule en décrivant une vaste courbe, qu'on appelle Les Échelles " (Constans, 1921, p. 202). La traduction de Clerc nous paraît préférable. Compte tenu de la position du trait de côte présumée ici, la distance donnée par Ammien est respectée.

$$
\text { * * }
$$

En éclairant les conditions naturelles dans le golfe de Fos à la fin de l'Âge du Fer et durant la période romaine, les études environnementales apportent des éléments décisifs à la compréhension du problème que nous évoquions dans l'introduction. Une grande mobilité caractérise l'histoire d'un littoral dont l'instabilité est accrue par les mouvements du sol (Vella, Bourcier, 1998). Durant le $\mathrm{I}^{\mathrm{er}}$ s. après J.-C., le régime du Rhône, dont la barre s'était relevée un siècle et demi ou deux siècles plus tôt, connaît une nouvelle modification et l'embouchure du fleuve redevient accessible à la navigation. Dans ce contexte, les variations des apports sédimentaires et des dynamiques marines observées dans le delta expliquent le creusement du canal de Marius, puis son abandon et les difficultés à le localiser.

Ces recherches confirment donc l'interprétation d'A. Grenier pour qui cet ouvrage avait mal fonctionné et avait été abandonné dès la fin du ${ }^{\text {er }}$ s. après $J$.C. au profit d'un accès par le bras principal du Rhône. En ce qui concerne sa localisation, on en distinguera les parties amont et aval. En aval, à la suite du recul survenu entre le $V^{c}$ et le $V_{I I}{ }^{c}$ s. après J.-C., l'érosion littorale a manifestement détruit les aménagements qui devaient en marquer le débouché maritime, et on ne peut plus s'étonner de ne pas les retrouver. Vers l'amont, il faut écarter l'hypothèse de l'existence d'un canal ou d'une lagune navigable dans le marais du Cavaou. L'orientation des lignes de rivage permet de déduire que, à l'est du delta, jusqu'au Moyen Âge, l'embouchure du Rhône reste celle d'Ulmet comme l'a démontré A. L'Homer, et qu'aucun bras du Rhône n'alimente directement le littoral de Fos. La recherche d'une structure excavée correspondant au canal de Marius doit donc se faire à partir du nouveau trait de côte proposé, c'est-à-dire légèrement au nord du Galéjon en direction du Rhône d'Ulmet ou directement d'Arles. 